Check for updates

Cite this: Chem. Sci., 2019, 10, 1332

๑ All publication charges for this article have been paid for by the Royal Society of Chemistry

Received 31st August 2018

Accepted 22nd November 2018

DOI: $10.1039 / \mathrm{c} 8 \mathrm{sc} 03897 \mathrm{j}$

rsc.li/chemical-science

\section{Mapping the polymorphic transformation gateway vibration in crystalline 1,2,4,5-tetrabromobenzene $\uparrow$}

\author{
Adam J. Zaczek, (D) a Luca Catalano, (D) ${ }^{\mathrm{b}}$ Panče Naumov (iD ${ }^{\mathrm{b}}$ \\ and Timothy M. Korter (D)*a
}

\begin{abstract}
The thermosalient behavior of 1,2,4,5-tetrabromobenzene (TBB) is related to a temperature-induced polymorphic structural change. The mechanism behind the phase transition has been investigated in this work using lowfrequency $\left(10-250 \mathrm{~cm}^{-1}\right)$ Raman spectroscopy and solid-state density functional theory simulations. Careful adjustments of the probing laser power permitted thermal control of the polymorph populations and enabled high-quality Raman vibrational spectra to be obtained for both the $\beta$ (low temperature) and $\gamma$ (high temperature) forms of TBB. Numerous well-defined vibrational features appear in the Raman spectra of both polymorphs which could be assigned to specific motions of the solid-state TBB molecules. It was discovered that the lowest-frequency vibration at $15.5 \mathrm{~cm}^{-1}$ in $\beta$-TBB at $291 \mathrm{~K}$ is a rotational mode that functions as a gateway for inducing the polymorphic phase transition to $\gamma$-TBB, and serves as the initiating step in the storage of mechanical strain for subsequent macroscopic release. Computationally mapping the potential energy surface along this vibrational coordinate reveals that the two TBB polymorphs are separated by a $2.40 \mathrm{~kJ} \mathrm{~mol}^{-1}$ barrier and that $\gamma$-TBB exhibits an enhanced cohesion energy that stabilizes its structure.
\end{abstract}

\section{Introduction}

The thermosalient effect is a phenomenon where a crystal undergoes a structural transformation due to changes in temperature, and this produces a physical "jumping" of the crystal. ${ }^{1-3}$ From an atom-level perspective, the physical motion corresponds with sudden yet small changes of the molecular structures and packing arrangements of the components comprising the crystalline solid. This unusual behavior has been attributed to material changes such as molecular dimerization (photosalient effect) ${ }^{4}$ and polymorph transformations ${ }^{5,6}$ (thermosalient effect). Given that this is often a reversible process ${ }^{7,8}$ controlled by varying sample temperature, the effect has attracted interest in the development of new energy transducing and actuating materials, such as those in electronics applications. ${ }^{9}$

One of the more well-studied thermosalient materials is 1,2,4,5-tetrabromobenzene (TBB). ${ }^{10-13}$ TBB is often used as a starting material in the organic syntheses of liquid crystals ${ }^{14}$ and photoconductive polymers. ${ }^{15}$ Below $307 \mathrm{~K}^{12,16}$ (previously reported at $319 \mathrm{~K}$ in ref. 17 and 18), crystalline TBB naturally exists in its $\beta$ polymorph, with the $\gamma$ polymorph forming at higher temperatures. Single crystals of TBB are often twinned, ${ }^{19,20}$ and the crystals jump or split upon polymorph transformation, propelling

\footnotetext{
${ }^{a}$ Department of Chemistry, Syracuse University, 1-014 Center for Science and Technology, Syracuse, New York, 13244-4100, USA. E-mail: tmkorter@syr.edu ${ }^{b}$ New York University Abu Dhabi, P.O. Box 129188, Abu Dhabi, United Arab Emirates $\dagger$ Electronic supplementary information (ESI) available. See DOI: $10.1039 / \mathrm{c} 8 \mathrm{sc} 03897 \mathrm{j}$
}

themselves several centimeters. The jumping distances are largely dependent on the crystal size and resting face of the crystal, with large crystals having more pronounced jumping. ${ }^{10}$

The structures of both TBB polymorphs have been previously determined through single-crystal X-ray diffraction, ${ }^{21,22}$ revealing that both exist in the same space group $\left(P 2_{1} / n\right)$ with similar unit cell dimensions, differing primarily through an intermolecular tilting of the TBB rings with respect to one another in the unit cell, as shown in Fig. 1. Because the polymorph structures are quite similar to one another and do not intuitively correspond to the significant structural changes that might be associated with thermosalient behavior, it warrants further analysis of the crystal structures, the intermolecular lattice vibrations, and the underlying mechanism responsible for the conversion between the solid forms.

Recent work has investigated the structural strain associated with the polymorph change that contributes to the thermosalient nature of TBB, and suggested that low-frequency vibrational modes near $43 \mathrm{~cm}^{-1}$ and $47 \mathrm{~cm}^{-1}$ are integral to the conversion. ${ }^{12}$ The importance of low-frequency vibrations for determining the properties of TBB crystals is consistent with the established relationship that exists between low-frequency lattice phonons and displacive/martensitic phase transformations as observed in other crystalline solids. ${ }^{23-25}$ In the current study, experimental lowfrequency Raman spectral data $\left(10-250 \mathrm{~cm}^{-1}\right)$ was collected at various temperatures and combined with solid-state density functional theory (ss-DFT) simulations to provide clear assignments of the vibrational motions in this region. This granted the ability to uniquely identify a single mode as being the gateway 

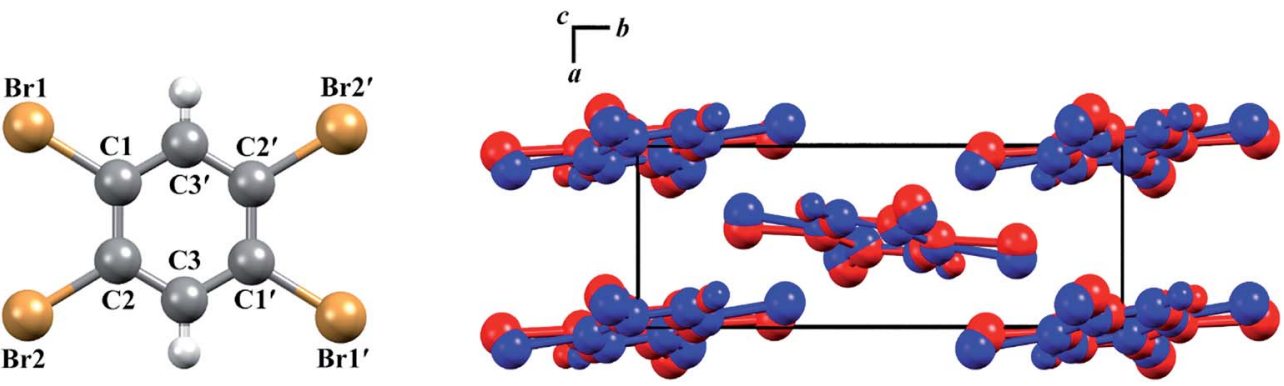

Fig. 1 Molecular structure of a TBB molecule (left) and the packing differences between the two TBB polymorphs, $\beta$-TBB (blue) and $\gamma$-TBB (red).

vibration for $\beta$-TBB to $\gamma$-TBB conversion. An in-depth computational study of the vibrational energies, motions, and potential energy surfaces of six rotational modes present in the $\beta$-ТВB crystals revealed that only the vibration observed at $15.5 \mathrm{~cm}^{-1}$ (at $291 \mathrm{~K}$ ) is able to initiate the $\beta \rightarrow \gamma$ transformation. The combination of experimental Raman data and ab initio computational analyses enables the mechanism of the polymorph transformation to be understood and quantified in terms of relative energies and barriers to conversion.

\section{Methods}

\section{a. Experimental details}

TBB was purchased from Sigma-Aldrich and used without further purification. Sample composition was confirmed via powder X-ray diffraction, showing $\beta$-TBB. Raman spectra were obtained with an Ondax (Monrovia, CA, USA) THz-Raman spectrometer with a laser centered at $784.7 \mathrm{~nm}$ and coupled to an Andor (Concord, MA, USA) Shamrock 750 spectrograph with an Andor iDus $416 \mathrm{CCD}$. The data spanned $\pm 250 \mathrm{~cm}^{-1}$ from the Rayleigh peak, with an effective spectral resolution of $0.6 \mathrm{~cm}^{-1}$. For room-temperature studies, the TBB sample was kept as a loose powder in a $10 \mathrm{~mm}$ diameter glass vial. The spectra shown here were averaged over 225 acquisitions, each with a 3 second exposure time. A range of laser power levels was used $(2.5,5.2,13.1,17.7,74$, and $115 \mathrm{~mW})$ and controlled by a variable neutral density filter.

For cooled studies, the powder samples were compressed into free-standing pellets (13 mm diameter, $\sim 1 \mathrm{~mm}$ thick) using pure TBB and mounted in a liquid-nitrogen-cooled cryostat. With an applied pelleting pressure of $0.07 \mathrm{GPa}$, the sample disc showed $\beta$-TBB to be the major constituent, but with $0.14 \mathrm{GPa}$ applied, $\gamma$-TBB was the major component. Additional pellets were made at increased pressures $(0.20$ and $0.28 \mathrm{GPa})$ to evaluate the influence on TBB stabilization and were examined only at $290 \mathrm{~K}$. The laser power was kept to a minimum $(13.1 \mathrm{~mW})$ for all cooled pellet experiments to avoid unwanted polymorph conversion while maximizing the scattering signal.

\section{b. Computational details}

All solid-state density functional theory (ss-DFT) calculations were performed using the CRYSTAL17 (ref. 26) software package. The Perdew-Burke-Ernzerhof (PBE) exchange and correlation functional ${ }^{27}$ was used with the def2-TZVP ${ }^{28}$ basis set.
Grimme's D3 dispersion correction ${ }^{29,30}$ with an included BeckeJohnson dampening parameter ${ }^{31}$ was employed to treat weak long-range London dispersion forces between molecules.

Geometry optimizations were begun using previously published crystal structures for $\beta-\mathrm{TBB}^{21}$ and $\gamma-\mathrm{TBB}^{22}$ as initial starting points. To account for the effects of non-zero experimental temperatures on the unit cell dimensions in the calculations, atomic positions were allowed to optimize to their energetic minima within fixed lattice dimensions and space group symmetries determined by the reported single-crystal X-ray diffraction measurements. For $\beta$-TBB (the low-temperature form), a free optimization that did not constrain lattice dimensions was also run for comparison. An energetic convergence of $\Delta E<10^{-8}$ hartree was used for all optimizations with root-meansquare thresholds (in atomic units) set to $1.0 \times 10^{-5}$ for the gradients and $4.0 \times 10^{-5}$ for the estimated displacements. Separate single-point energy calculations were performed on the optimized structures in order to determine the energy stability rankings of the polymorphs, and these were corrected for basis set superposition errors (BSSE) using the counterpoise method. ${ }^{32}$

Harmonic normal mode frequency analyses were executed on the structurally optimized TBB solids with a stricter energy convergence of $\Delta E<10^{-10}$ hartree. Raman intensities were calculated by computing the Raman tensor utilizing a coupledperturbed Hartree-Fock/Kohn-Sham approach. ${ }^{33,34}$ To accelerate convergence, a mixing of Fock/KS matrix derivatives ${ }^{35}$ was used. Experimental temperatures and laser excitation wavelength were also accounted for in the simulated intensities. Normal mode analyses formed the basis for constrained unit cell optimizations, using eigenvector-displaced molecular positions to determine the influence of specific lattice vibrations on the polymorphic stabilities.

For all calculations, truncation tolerances for the Coulomb and Hartree-Fock exchange integrals (keyword TOLINTEG) were set to $10^{-12}, 10^{-12}, 10^{-12}, 10^{-24}, 10^{-48}$ hartree. A sampling of $205 k$-points was used in the irreducible Brillouin zone (keyword SHRINK $=9$ ), and a predefined grid size of 366477 points was utilized (keyword XXLGRID).

\section{Results and discussion}

\section{a. Raman spectroscopy of powder samples}

Raman spectra of TBB powder were taken using a $784.7 \mathrm{~nm}$ laser with varying power levels, with the spectrum at the lowest 
power $(2.5 \mathrm{~mW})$ showing $\beta$-TBB, and the maximum power (115 $\mathrm{mW}$ ) revealing $\gamma$-TBB to be present (Fig. 2). The spectral features of both forms were consistent with prior reports, ${ }^{36,37}$ aiding in their identification. The samples at the laser power extremes were found to be at least $96 \%$ polymorphically pure within the limits of detection. Polymorph changes occurred due to laser heating of the sample, with greater powers imparting more heat and causing $\gamma$-TBB formation. At intermediate powers, a mix of both polymorphs was observed, which may be attributed to uneven sample heating leading to localized partial polymorph conversion in the beam path. After approximately $35.0 \mathrm{~mW}, \gamma$ TBB was observed as the clear majority product. No thermosalient crystal movement was observed in the powder sample during heating (likely due to the very small particle size), indicating that the spectra were representative of the same probed area each time.

It is possible to calculate the average temperature $(T)$ of a sample at the laser focus directly from the Raman spectral data by utilizing the relative intensities of the anti-Stokes/Stokes scattering features and the following equation:

$$
\frac{I_{\text {anti-Stokes }}}{I_{\text {Stokes }}}=\left(\frac{\nu_{0}+\nu_{1}}{\nu_{0}-\nu_{1}}\right)^{4} \mathrm{e}^{\frac{-h \nu_{1}}{k T}}
$$

where $I$ values originate from the intensities of an anti-Stokes and Stokes peak pair, $\nu_{0}$ is the frequency of the exciting light, $\nu_{1}$ is the frequency of the Raman shift, $h$ is Planck's constant, and $k$ is the Boltzmann constant. Temperatures were calculated using the spectral features at $\pm 126.4 \mathrm{~cm}^{-1}$ from the Rayleigh peak, as these are single narrow features that are present in both TBB polymorphs, as shown in Fig. 3. The peaks were fit with a Lorentzian line shape, and the areas under the best fit curves were used in the anti-Stokes/Stokes ratio. All sample temperatures reported in this work have been determined in this way. The lowest applied power of $2.5 \mathrm{~mW}$ was found to produce a sample temperature of $291 \pm 3 \mathrm{~K}$, which is in agreement with the laboratory temperature of $290 \mathrm{~K}$, and showed the spectrum of $\beta$-TBB. The highest laser power data $(115 \mathrm{~mW})$ showed that the sample had been heated to $318 \pm 7 \mathrm{~K}$ and revealed the Raman spectrum of $\gamma$-TBB, in agreement with previous reports noting its dominance above $307 \mathrm{~K}^{\mathbf{1 2}}$

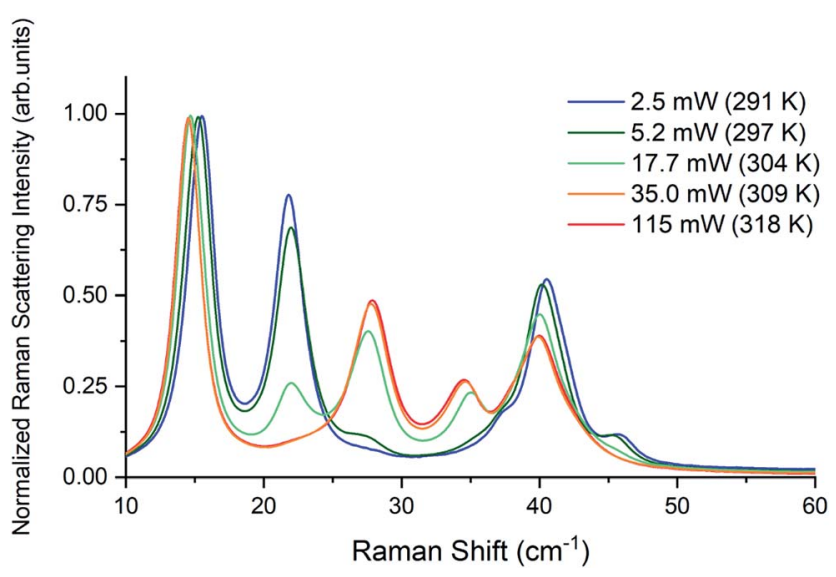

Fig. 2 Low-frequency Raman spectra of TBB at varying applied laser powers.

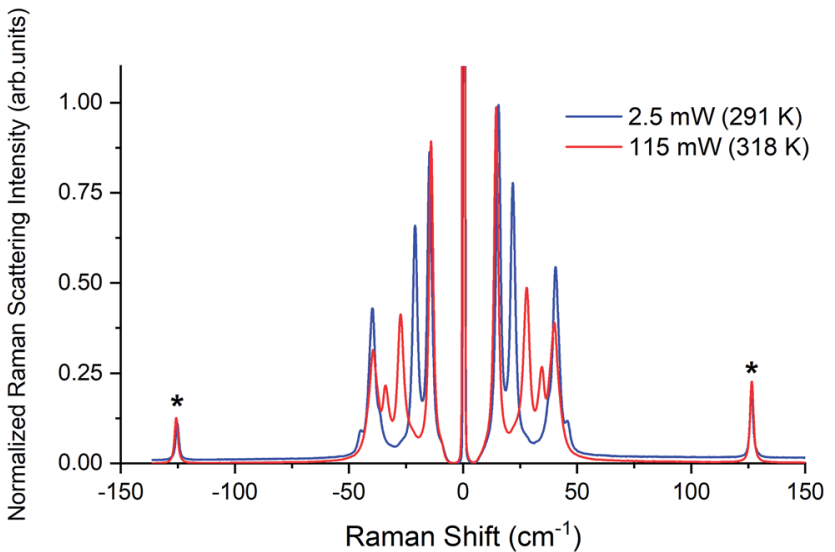

Fig. 3 Raman spectra of $\beta$-TBB (blue) and $\gamma$-TBB (red) obtained from powder samples with peaks used for temperature analysis marked by asterisks (*).

\section{b. Raman spectroscopy of pelleted samples}

i. $\boldsymbol{\beta}$-TBB. A $\beta$-TBB pellet made from the application of $0.07 \mathrm{GPa}$ was placed in a cryostat and studied both with liquidnitrogen cooling and with no cooling. A low laser power (13.1 $\mathrm{mW}$ ) was used in all cases to record the Raman spectra shown in Fig. 4 and at-sample temperatures of $317 \pm 6 \mathrm{~K}$ (uncooled) and $105 \pm 4 \mathrm{~K}$ (cooled) were determined. The pellet spectra match with the corresponding Raman spectra of the powder samples, with the only differences (referring to the $105 \mathrm{~K}$ data) being a shoulder feature at $14.8 \mathrm{~cm}^{-1}$ and a peak at $32.2 \mathrm{~cm}^{-1}$ originating from a small amount of coexisting $\gamma$-TBB. The $\gamma$-TBB contributions have been identified through a direct comparison of the pellet data over a range of temperatures and compared to TBB powder samples (data provided in the ESI $\dagger$ ). The unexpected presence of $\gamma$-TBB at $105 \mathrm{~K}$ may be due to transient heating upon pressure application during sample preparation, but it may also be from pressure-induced formation given that $\gamma$-TBB has been reported to be stable in nanopore structures. ${ }^{10}$ Similarly, the observation of the clear Raman features from $\beta$-TBB at $317 \mathrm{~K}$ is inconsistent with the reported transition temperature of $307 \mathrm{~K}$,

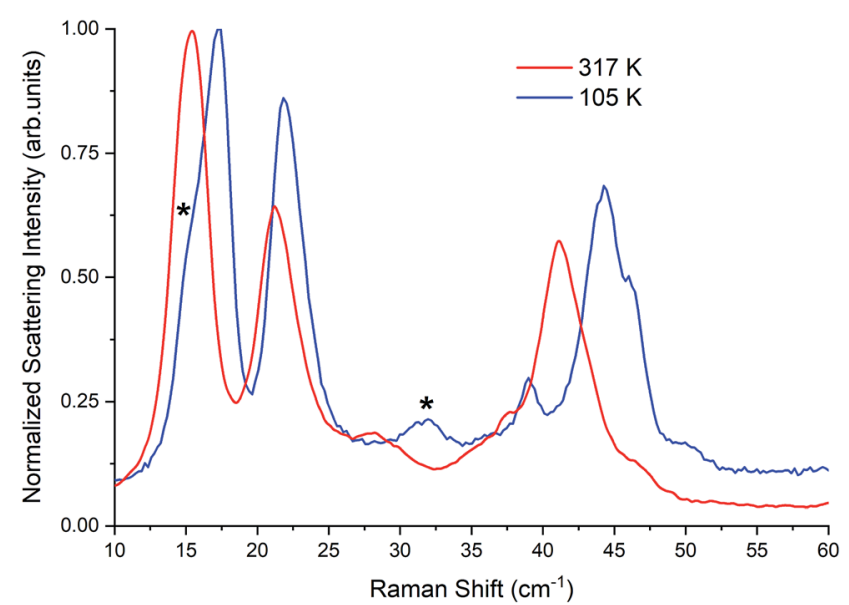

Fig. 4 Raman spectrum of $\beta$-TBB at $317 \mathrm{~K}$ and $105 \mathrm{~K}$. Contributions from $\gamma$-TBB in the $105 \mathrm{~K}$ spectrum are marked with asterisks $(*)$. 
but it is apparently stabilized by the applied pelleting pressure. Using a linear combination of the powder spectra, it was found that the sample pellet created at $0.07 \mathrm{GPa}$ contained approximately $90 \% \beta$-TBB and 10\% $\gamma$-TBB. While the presence of $\gamma$-TBB was initially undesirable, this result presented an opportunity since this demonstrated it could be stabilized and studied simultaneously with $\beta$-TBB at low temperatures.

ii. $\gamma$-TBB. To further test the stabilization of $\gamma$-TBB with pressure, another pellet was made by compressing to $0.14 \mathrm{GPa}$ in an attempt to create a pellet with a higher $\gamma$-TBB concentration. The resulting sample was found to contain $80 \% \gamma$-TBB, with the remainder being $\beta$-TBB. Further evidence that these peaks correspond to $\beta$-TBB is demonstrated in spectra at intermediate temperatures, provided in the ESI. $\dagger$ Remarkably, when this pellet was cooled to cryogenic temperatures (well below the temperature that $\gamma$-TBB should be stable), the spectral features indicated that no conversion to $\beta$-TBB had occurred. The temperature-dependent spectra for $\gamma$-TBB at 320 $\pm 7 \mathrm{~K}$ and $118 \pm 4 \mathrm{~K}$ are shown in Fig. 5 . This is the first time that $\gamma$-TBB has been stabilized at this low a temperature, although recent work has shown that $\gamma$-TBB nanocrystals have been stabilized in anodic aluminum oxide nanopores to $183 \mathrm{~K}^{.10}$

Pressure clearly plays a role in TBB polymorph stabilization. To investigate this, additional pellets were made at varying pressures $(0.03,0.10,0.20$, and $0.28 \mathrm{GPa})$. While very little change was observed in the room-temperature Raman spectra at intermediate pressures, the $0.28 \mathrm{GPa}$ pellet was found to contain an additional 5\% $\gamma$-TBB (85\% in total). Although this did not eliminate the $\beta$-TBB form completely, it showed that pressure increases do promote $\gamma$-TBB stabilization. It is worth noting that higher pressures $(0.7 \mathrm{GPa})$ can cause formation of $\alpha$ TBB,${ }^{38}$ but the pressures used in the present study were not great enough to observe any trace of this polymorph.

\section{c. Solid-state density functional theory simulations}

i. Structural optimizations. ss-DFT simulations were initially performed on the $\beta$-TBB polymorph. A full optimization of the

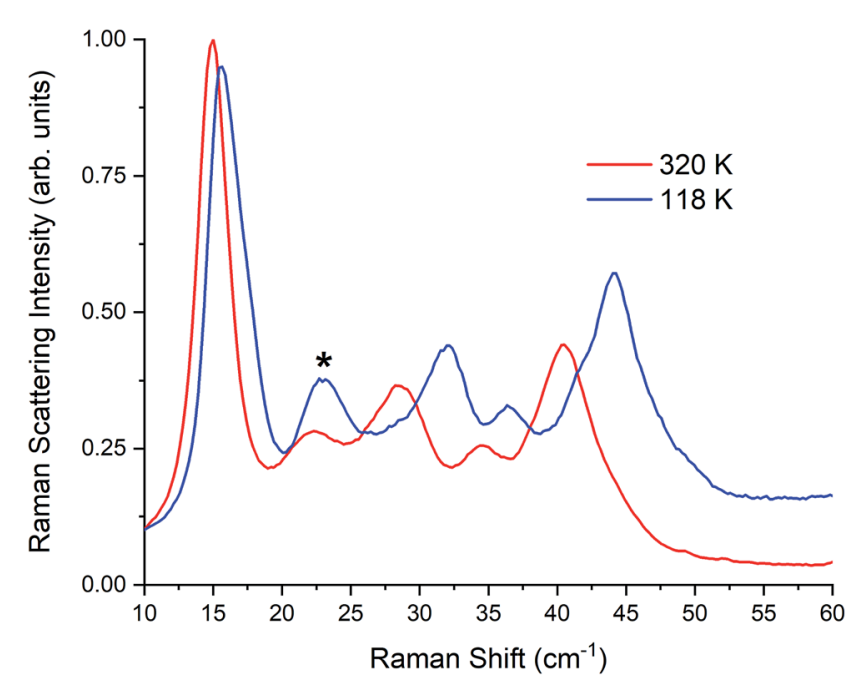

Fig. 5 Raman spectra of $\gamma$-TBB at $320 \mathrm{~K}$ and $118 \mathrm{~K}$. Contribution from $\beta$-TBB is marked in the $118 \mathrm{~K}$ spectrum with an asterisk (*). unit cell dimensions and atomic positions produced excellent results (Table 1), with errors $\leq 0.15 \%$ in all cell parameters. However, when full optimizations were performed on $\gamma$-TBB, the structure immediately relaxed to the $\beta$-TBB form. The ssDFT simulations are performed at $0 \mathrm{~K}$ where $\beta$-TBB is experimentally known to be more stable, so the conversion is not surprising. The two polymorphs exhibit the same space group symmetry $\left(P 2_{1} / n\right)$ and differ only by a small intermolecular tilting of the rings. However, it is important to note that the $\gamma$ TBB crystal maintains its form and optimizes without issue (including having all positive frequency eigenvalues) if the unit cell volume is constrained and held constant at the $332 \mathrm{~K}$ experimental value. This indicates that both polymorph structures represent minima on the solid-state potential energy surface (at least in terms of enthalpy), but that thermal expansion and contraction of the unit cell plays an important role in defining polymorph stabilities.

Given these results, optimization of $\gamma$-TBB was done with crystal lattice parameters held at experimentally observed values ( $332 \mathrm{~K}$ (ref. 22)) and the atoms were allowed to relax to an energetic minimum within these constraints. While no problems were encountered in the full optimization of the $\beta$-TBB structure, for a fair comparison to the fixed-lattice $\gamma$-TBB calculations, a fixed-lattice (100 K (ref. 21)) optimization was also performed on $\beta$-TBB. All energy and vibrational analyses presented in this work are based on the fixed-lattice calculations. Regardless of the constraints on the geometry optimizations, the molecular structures produced in the simulations agreed very well with experimental data, as shown by the calculated intramolecular distances and angles, as well as the intermolecular distances, shown in Table 2.

ii. Vibrational analysis. Simulations of the Raman spectra of both TBB polymorphs were in excellent agreement with experimental observations (Fig. 6) and no scaling of the vibrational frequencies was applied. A complete list of all calculated vibrational modes is available in the ESI. $\dagger$ The $\beta$-TBB and $\gamma$-TBB simulations were compared to their corresponding $105 \mathrm{~K}$ and $320 \mathrm{~K}$ spectra, respectively, as these were closest to the temperatures of the reported crystallographic data. All Ramanactive modes below $100 \mathrm{~cm}^{-1}$ represent rigid rotations of the TBB molecules in their lattice positions. This was determined through analysis of the normal mode eigenvector displaced structures, which yielded no significant changes in the intramolecular geometries of the TBB molecules (see ESI $\dagger$ ). The peak at $126.4 \mathrm{~cm}^{-1}$ which had negligible shifting experimentally corresponds to an in-plane intramolecular bromine wagging (calculated at $125.6 \mathrm{~cm}^{-1}$ and $124.7 \mathrm{~cm}^{-1}$ in $\beta$-TBB and $\gamma$-TBB, respectively), while the peaks in the $190-250 \mathrm{~cm}^{-1}$ region originate from intramolecular ring torsions.

Complete lists of the experimental and calculated Ramanactive modes for both TBB polymorphs below $250 \mathrm{~cm}^{-1}$ are shown in Tables 3 and 4 . The $\beta$-TBB vibrational simulations are in excellent agreement with the current experimental results, with the only peaks not modeled arising from the aforementioned $\gamma$-TBB content. A recent study has presented a similar vibrational analysis of $\beta$-TBB, ${ }^{12}$ but it did not experimentally access the significant sub- $40 \mathrm{~cm}^{-1}$ range. The peaks calculated 
Table 1 Comparison of the measured $\beta$-TBB crystalline dimensions and results from a fully optimized PBE-D3/def2-TZVP structure

\begin{tabular}{llccrc}
\hline & $a(\AA)$ & $b(\AA)$ & $c(\AA)$ & $\beta\left(^{\circ}\right)$ & Volume $\left(\AA^{3}\right)$ \\
\hline Experimental $^{21}(100 \mathrm{~K})$ & 3.9235 & 10.4885 & 10.3675 & 100.367 & 419.675 \\
Calculated $_{\text {Error }(\%)}$ & 3.9241 & 10.5012 & 10.3559 & 100.516 & 419.580 \\
& 0.02 & 0.12 & -0.11 & 0.15 & -0.02 \\
\hline
\end{tabular}

for $\gamma$-TBB are also in excellent agreement with experimental results, with all features accounted for.

iii. Polymorph stabilities. The results of energetic calculations revealed that $\beta$-TBB is $0.79 \mathrm{~kJ} \mathrm{~mol}^{-1}$ (per molecule) lower in total electronic energy than $\gamma$-TBB. Inclusion of zero-point energies from the vibrational simulations did not change this rank, with $\beta$-TBB remaining the more stable polymorph by $0.75 \mathrm{~kJ} \mathrm{~mol}^{-1}$ at $0 \mathrm{~K}$. This is consistent with the observed dominance of $\beta$-TBB at temperatures below $307 \mathrm{~K}$. Additional insight into the relative stabilities can be gained by decomposing the total energy into its conformational and cohesive components, which are listed in Table 5. $\beta$-TBB was found to have a more stable conformational energy, while $\gamma$-TBB had a more favorable cohesive energy.

Temperature-dependent Gibbs free energy curves (also corrected for BSSE) were constructed for both polymorphs. $\beta$-TBB is more stable at $0 \mathrm{~K}$ by $0.75 \mathrm{~kJ} \mathrm{~mol}^{-1}$, but the curves cross at $76 \mathrm{~K}$ and $\gamma$-TBB becomes the more stable polymorph. While the transition temperature is quantitatively incorrect, it qualitatively agrees with experimental observations of $\gamma$-TBB being favored at higher temperatures.

iv. Normal mode eigenvector analysis. To examine the behavior of individual vibrations, the sub- $100 \mathrm{~cm}^{-1}$ modes in the Raman spectra were further analyzed using ss-DFT to construct potential energy surfaces associated with their motions. The modes from the simulated spectra at 18.7, 40.7, and $45.0 \mathrm{~cm}^{-1}$ represent three different molecular rigid rotations within the $\beta$-TBB crystal about the crystallographic $a, b$, and $c$ axes. The modes at 23.9, 52.3, and $45.5 \mathrm{~cm}^{-1}$ represent the same types of molecular motions, but with out-of-phase relationships between the members of the $Z=2$ crystallographic unit cell, instead of the in-phase relationship found in the previous modes.

In order to test which (if any) of the modes were responsible for polymorph conversion, the atoms in the optimized $\beta$-TBB structure were incrementally displaced along the rotational mode eigenvectors and the unit cell was optimized around the new structure. The results of these eigenvector scans are shown in Fig. 7 and reveal that both the $18.7 \mathrm{~cm}^{-1}$ mode and the $23.9 \mathrm{~cm}^{-1}$ mode displaced structures optimized towards $\gamma$-TBB unit cell dimensions, with the $18.7 \mathrm{~cm}^{-1}$ mode results noticeably closer to the known $\gamma$-TBB values.

By examining the eigenvector displaced crystals, it was found that the structure resulting from displacement along the $18.7 \mathrm{~cm}^{-1}$ eigenvector coordinate correlated very well with the atomic positions found in $\gamma$-TBB. This was not true for the outof-phase $23.9 \mathrm{~cm}^{-1}$ mode, which forced TBB to an unstable high energy arrangement that did not correspond to either TBB

Table 2 Comparison of the experimental and calculated bond lengths $(\AA)$, intramolecular angles $\left(^{\circ}\right)$, dihedral angles $\left(^{\circ}\right)$, and intermolecular distances $(\AA)$ involving non-hydrogen atoms for $\beta$-TBB and $\gamma$-TBB

\begin{tabular}{|c|c|c|c|c|}
\hline \multirow[b]{2}{*}{ Intramolecular bond $(\AA)$} & \multicolumn{2}{|l|}{$\beta$-TBB } & \multicolumn{2}{|l|}{$\gamma$-TBB } \\
\hline & Experimental & Calculated & Experimental & Calculated \\
\hline $\mathrm{C} 1-\mathrm{C} 2$ & 1.397 & 1.401 & 1.358 & 1.371 \\
\hline $\mathrm{C} 1-\mathrm{C} 3^{\prime}$ & 1.401 & 1.396 & 1.420 & 1.406 \\
\hline $\mathrm{C} 2-\mathrm{C} 3$ & 1.387 & 1.395 & 1.372 & 1.399 \\
\hline $\mathrm{C} 1-\mathrm{Br} 1$ & 1.884 & 1.895 & 1.844 & 1.873 \\
\hline $\mathrm{C} 2-\mathrm{Br} 2$ & 1.883 & 1.896 & 1.917 & 1.904 \\
\hline \multicolumn{5}{|l|}{ Angle $\left({ }^{\circ}\right)$} \\
\hline $\mathrm{C} 1-\mathrm{C} 2-\mathrm{C} 3$ & 121.82 & 121.74 & 121.74 & 121.82 \\
\hline $\mathrm{Br} 2-\mathrm{C} 2-\mathrm{C} 3$ & 118.15 & 118.34 & 118.34 & 118.15 \\
\hline $\mathrm{C} 1-\mathrm{C} 2-\mathrm{Br} 2$ & 120.03 & 121.74 & 121.73 & 120.03 \\
\hline Br1-C1-C2 & 119.84 & 119.63 & 119.63 & 119.84 \\
\hline \multicolumn{5}{|l|}{ Dihedral angle $\left({ }^{\circ}\right)$} \\
\hline $\mathrm{C} 3-\mathrm{C} 1-\mathrm{C} 2-\mathrm{C} 3$ & 0.07 & 0.10 & 1.67 & 0.03 \\
\hline $\mathrm{Br} 1-\mathrm{C} 2-\mathrm{C} 1-\mathrm{C} 3$ & 179.58 & 179.53 & 177.74 & 179.48 \\
\hline $\mathrm{Br} 1-\mathrm{C} 1-\mathrm{C} 2-\mathrm{Br} 2$ & 0.36 & 0.55 & 1.07 & 0.87 \\
\hline $\mathrm{Br} 2-\mathrm{C} 1-\mathrm{C} 2-\mathrm{C} 3$ & 180.00 & 179.98 & 178.15 & 179.63 \\
\hline \multicolumn{5}{|l|}{ Intermolecular bond (£) } \\
\hline $\mathrm{Br} 1 \cdots \mathrm{C} 3$ & 3.740 & 3.734 & 4.158 & 4.182 \\
\hline $\mathrm{Br} 2 \cdots \mathrm{C} 3$ & 4.477 & 4.469 & 4.129 & 4.154 \\
\hline
\end{tabular}



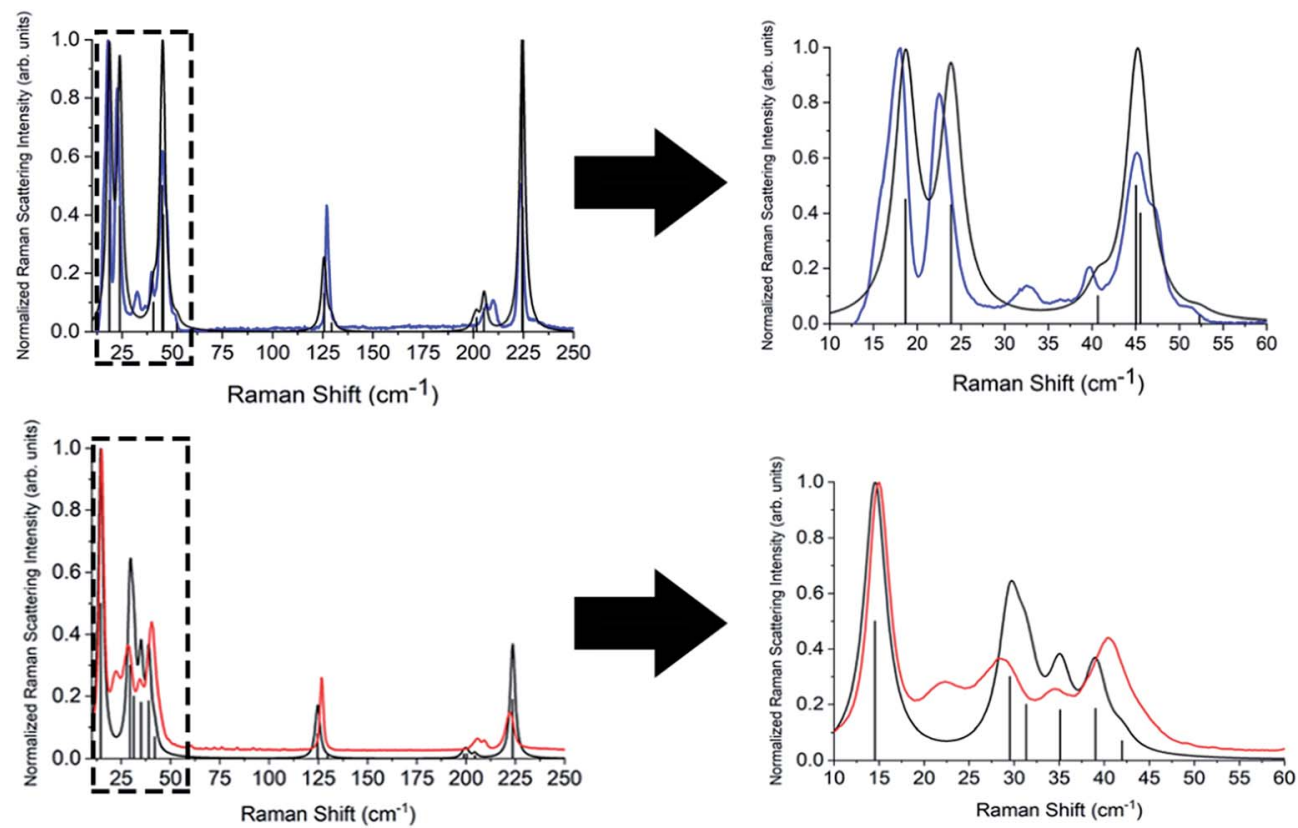

Fig. 6 Experimental Raman spectra for $\beta$-TBB (blue) at $107 \mathrm{~K}$ and $\gamma$ (red) at $318 \mathrm{~K}$ with their corresponding simulated (black) Raman spectra. The boxed areas in the lower frequency range (left) are shown expanded on the right.

polymorph. Although it appears from Fig. 7 that the $23.9 \mathrm{~cm}^{-1}$ mode goes towards $\gamma$-TBB formation, the actual atomic positioning contradicts this, and only the $18.7 \mathrm{~cm}^{-1}$ mode yields both unit cell dimensions and atomic positions that match those of $\gamma$-TBB. The vibrational motion of the $18.7 \mathrm{~cm}^{-1}$ mode is represented in Fig. 8.

A less strained and therefore more realistic approach for mapping the potential energy surface of the polymorph transformation coordinate is to freeze a single coordinate and then step along it, allowing the rest of the system to fully relax. A change in the intermolecular $\mathrm{C} 2-\mathrm{Br} 2 \cdots \mathrm{C} 3^{\prime}-\mathrm{C} 1$ dihedral angle mimics the TBB ring tilting differences between the polymorphs that can be seen in Fig. 1 and this dihedral angle also corresponds to the rotational motion that forms the basis of the $18.7 \mathrm{~cm}^{-1}$ vibration.

Table 3 Experimental and simulated Raman spectral features (in $\mathrm{cm}^{-1}$ ) of $\beta$-TBB with comparison to published observations. Shoulder features are indicted with "sh". Calculated intensities have been normalized to 1 in this spectral range

\begin{tabular}{|c|c|c|c|c|c|c|}
\hline \multirow{2}{*}{$\begin{array}{l}\text { White, et al. }{ }^{36} \\
\text { Experimental } \\
\text { (ambient) }\end{array}$} & \multirow{2}{*}{$\begin{array}{l}\text { Zakharov, et al. }^{12} \\
\text { Experimental } \\
\text { (ambient) }\end{array}$} & \multirow{2}{*}{$\begin{array}{l}\text { Burgos \& Bonadeo }^{37} \\
\text { Experimental } \\
(100 \mathrm{~K})\end{array}$} & \multicolumn{4}{|l|}{ Current work } \\
\hline & & & $\begin{array}{l}\text { Experimental } \\
(291 \mathrm{~K})\end{array}$ & Experimental (105 K) & $\begin{array}{l}\text { Calculated (100 } \\
\text { K lattice) }\end{array}$ & $\begin{array}{l}\text { Calculated } \\
\text { intensities }\end{array}$ \\
\hline - & - & 17.5 & 15.5 & 17.1 & 18.7 & 0.969 \\
\hline - & - & 21.5 & 21.8 & 22.1 & 23.9 & 0.923 \\
\hline- & - & 38.5 & - & 39.0 & 40.7 & 0.105 \\
\hline - & 45.5 & 50.5 & 46.2 & 50.1 & 52.3 & 0.025 \\
\hline - & - & 52 & - & - & - & - \\
\hline - & 54 & - & - & - & - & - \\
\hline & - & 62.0 & - & - & - & - \\
\hline - & 87 & - & - & - & - & - \\
\hline 125 & 126.4 & - & 126.4 & 126.6 & 125.6 & 0.312 \\
\hline 208 & 209 & - & & & 205.5 & 0.127 \\
\hline 220 & 221 & - & 221.4 & 223.4 & 224.4 & 1.000 \\
\hline- & - & - & - & $224.5(\mathrm{sh})$ & 224.7 & 0.427 \\
\hline- & 234 & - & - & - & - & - \\
\hline
\end{tabular}


Table 4 Experimental and simulated Raman spectral features (in $\mathrm{cm}^{-1}$ ) of $\gamma$-TBB with comparison to published observations. Calculated intensities have been normalized to 1 in this spectral range

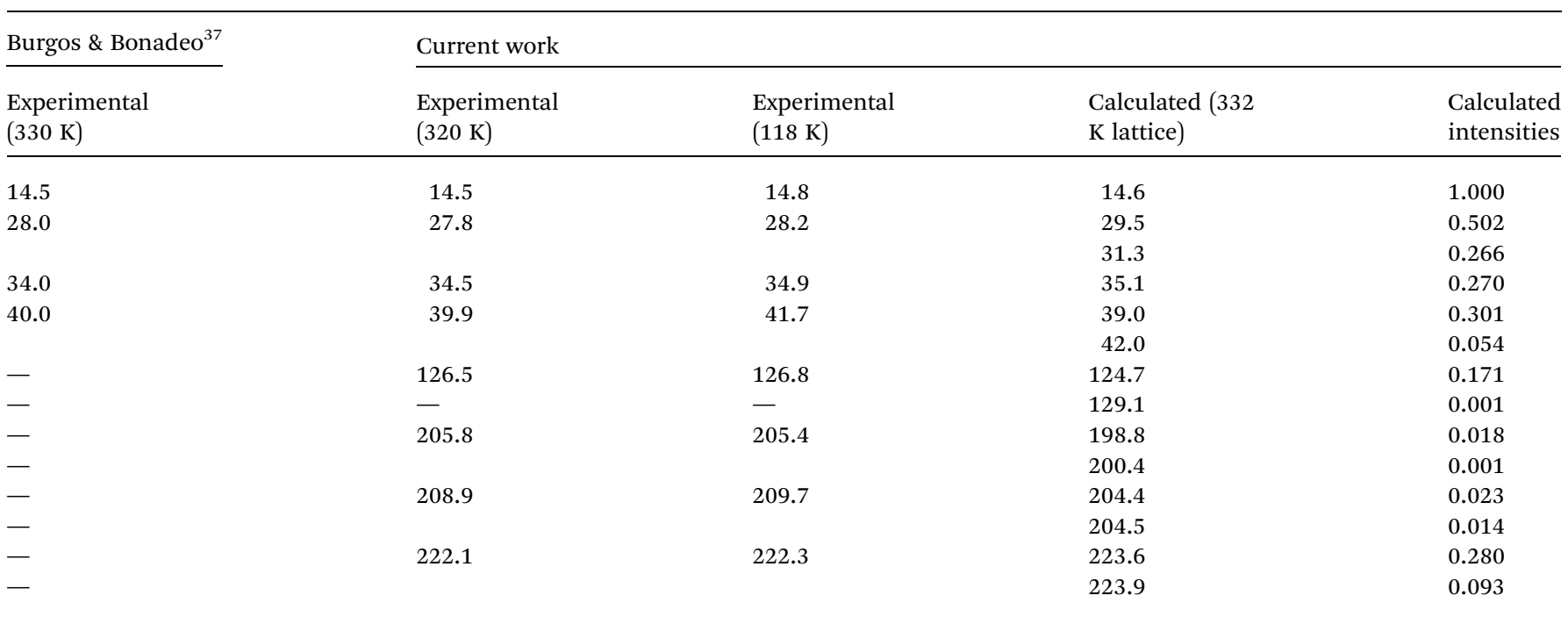

Table 5 Calculated total electronic, conformational, and cohesive energies of the TBB polymorphs. All energies are in $\mathrm{kJ} \mathrm{mol}^{-1}$ per molecule and corrected for BSSE

\begin{tabular}{llll}
\hline & $\begin{array}{l}\text { Total energy } \\
\text { (relative) }\end{array}$ & $\begin{array}{l}\text { Conformational } \\
\text { energy (relative) }\end{array}$ & Cohesive energy \\
\hline$\beta$-TBB & 0 & 0 & -144.854 \\
$\gamma$-TBB & 0.791 & 2.111 & -146.174
\end{tabular}

Optimizations were performed by initially freezing the dihedral value at $10.5^{\circ}$, corresponding to the angle observed in a full optimization of $\beta$-TBB, and incrementally increasing the angle towards the $\gamma$-TBB polymorph. All other atomic positions and lattice parameters were allowed to energetically relax. A second energy minimum was found for the structure at a dihedral angle of $25.3^{\circ}$, which is identical to the dihedral angle observed in $\gamma$-TBB.

Although the unit cell dimensions and atomic positions are in good agreement with the $\gamma$-TBB structure at this dihedral angle, there was a $4.6 \%$ contraction of the cell volume as compared to experiment. ${ }^{22}$ However, contraction is expected in a $0 \mathrm{~K}$ structural simulation (especially versus the $332 \mathrm{~K}$ experimental data $^{22}$ ) and the volume reduction is not unlike that reported for $\beta$-TBB when comparing room temperature to $100 \mathrm{~K}$ (ref. 21) structures, which is about $5.0 \%$.

The energy values determined over the course of the dihedral scanning are shown in Fig. 9. This revealed a barrier height of $2.40 \mathrm{~kJ} \mathrm{~mol}^{-1}$, which is a reasonable energy for the $\beta \rightarrow \gamma$ polymorph transformation considering the reported transition temperature of $307 \mathrm{~K}$ (ref. 12) (2.55 kJ mol${ }^{-1}$ ). Performing a similar analysis on the dihedral angle changes that corresponded to the $23.9 \mathrm{~cm}^{-1}$ mode only resulted in an increase in energy along the potential energy scan, with no indication of $\gamma$ TBB formation. As a final check to ensure that the dihedral constraint placed on the $\gamma$-TBB did not cause chemically
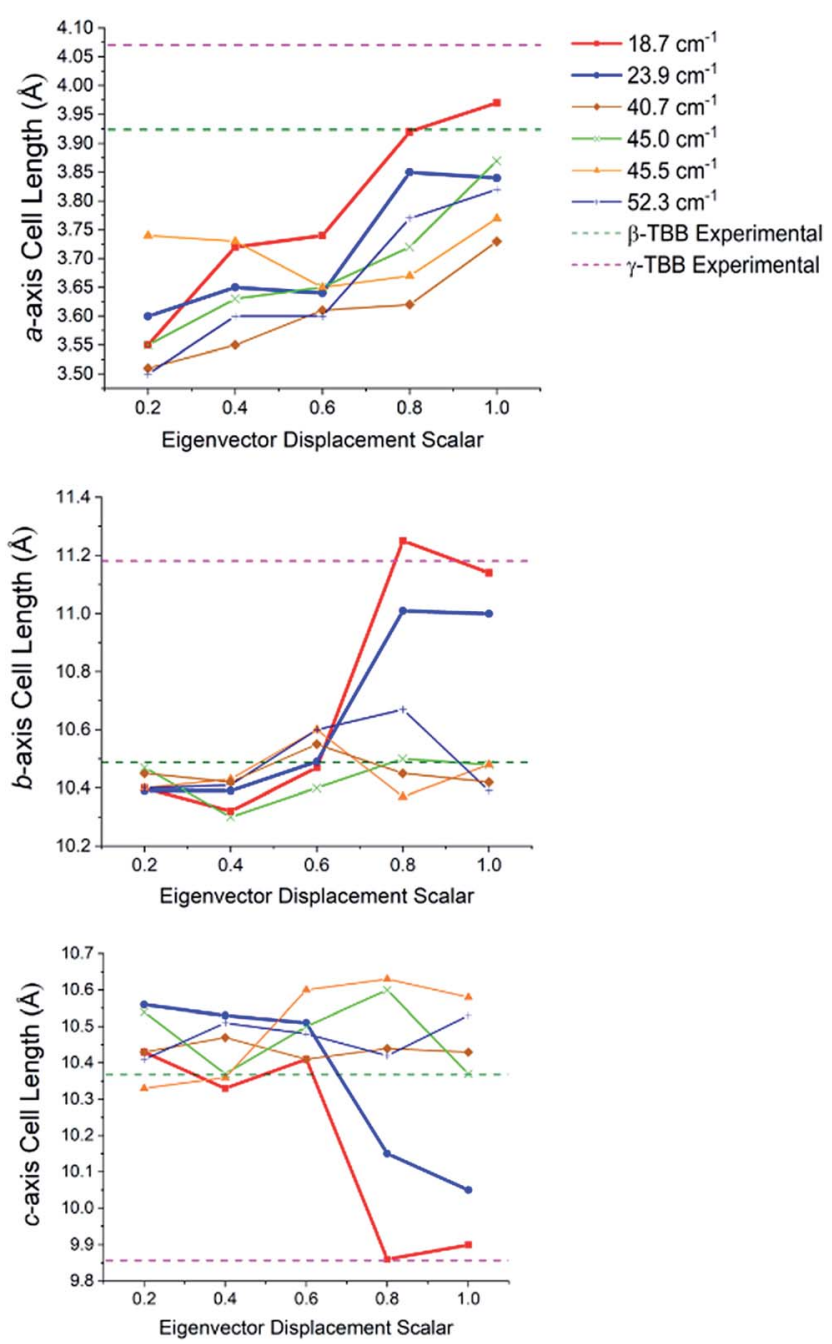

Fig. 7 Changes in the lattice dimensions of $\beta$-TBB resulting from displacement along rotational-type lattice vibrations. 

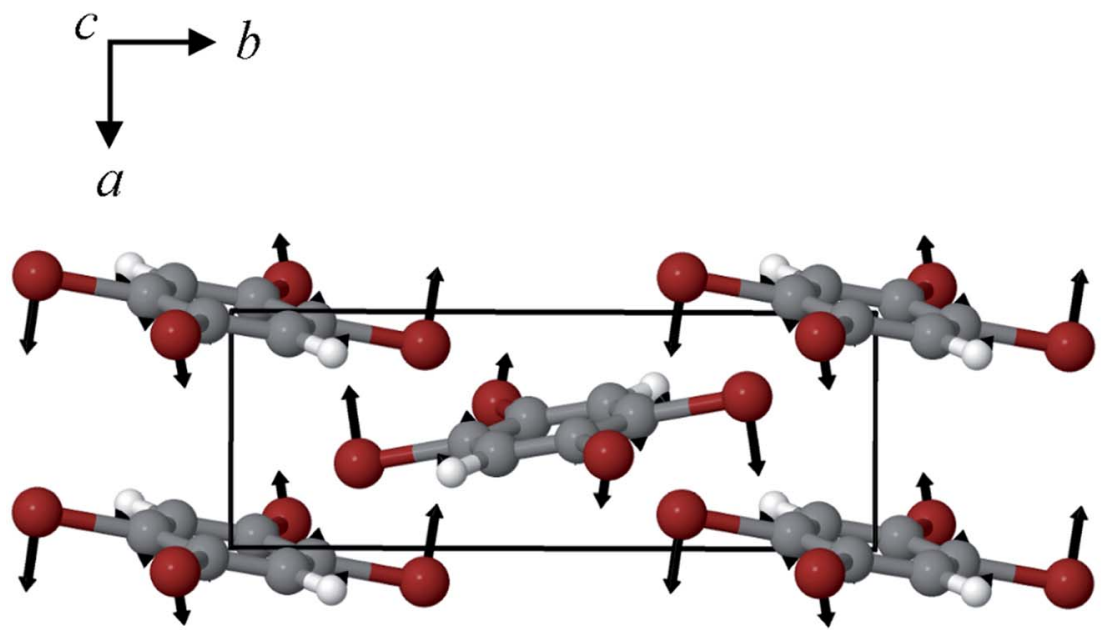

Fig. 8 Calculated eigenvector representation of the $18.7 \mathrm{~cm}^{-1}$ vibrational mode in $\beta$-TBB.

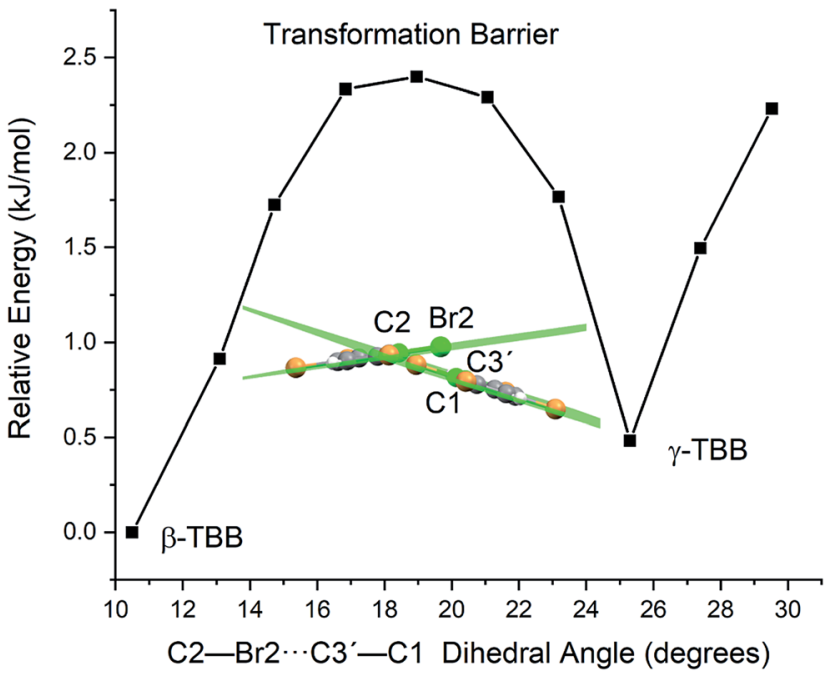

Fig. 9 Potential energy surface for a frozen dihedral scan corresponding to the calculated $18.7 \mathrm{~cm}^{-1}$ mode.

unrealistic approximations, a frequency analysis was performed and yielded no imaginary modes. These results collectively validate the dihedral-locked $\gamma$-TBB model solid to be a physically reasonable structure, and that the $18.7 \mathrm{~cm}^{-1}$ mode (observed experimentally at $17.1 \mathrm{~cm}^{-1}$ at $105 \mathrm{~K}$ and $15.5 \mathrm{~cm}^{-1}$ at $291 \mathrm{~K}$ ) is the sole gateway lattice vibration responsible for polymorph conversion.

\section{Conclusions}

A combined experimental and computational investigation of the Raman spectra and solid-state vibrational modes of crystalline TBB has revealed the specific vibrational mode responsible for polymorph conversion between the $\beta$ and $\gamma$ polymorphs. The solid-state DFT simulations were in excellent agreement with the experimental measurements, allowing for a very accurate assessment of the vibrational modes. By considering the rotational-type molecular motions and scanning along their eigenvectors in $\beta$ TBB, it was found that the $17.1 \mathrm{~cm}^{-1}$ mode in the $105 \mathrm{~K}$ Raman spectrum (calculated at $18.7 \mathrm{~cm}^{-1}$ and experimentally observed at $15.5 \mathrm{~cm}^{-1}$ at $291 \mathrm{~K}$ ) was able to act as a gateway vibration and induce the transformation of the $\beta$-form to the $\gamma$-form. This investigation also determined the barrier to conversion to be $2.40 \mathrm{~kJ} \mathrm{~mol}^{-1}$, which is consistent with the mild thermal energy required to form the $\gamma$-TBB polymorph. As suggested by previous reports, ${ }^{6,12}$ motion along this low-energy vibrational coordinate is translated into a macroscopic mechanical effect due to a combination of structural elasticity, anisotropic strain accompanying the transition, and the consequent stressed domain boundaries between $\beta$-form and $\gamma$-form domains within the crystal lattice. The discovery and characterization of the gateway vibration that is the foundation of polymorphic change in crystalline TBB offers new insights into its unusual thermosalient behavior and presents a compelling target of study in other materials that undergo temperature-induced polymorph changes.

\section{Conflicts of interest}

There are no conflicts to declare.

\section{References}

1 S. C. Sahoo, M. K. Panda, N. K. Nath and P. Naumov, Biomimetic crystalline actuators: Structure-kinematic aspects of the self-actuation and motility of thermosalient crystals, J. Am. Chem. Soc., 2013, 135(33), 12241-12251.

2 P. Commins, I. T. Desta, D. P. Karothu, M. K. Panda and P. Naumov, Crystals on the move: mechanical effects in dynamic solids, Chem. Commun., 2016, 52(97), 13941-13954.

3 P. Naumov, S. Chizhik, M. K. Panda, N. K. Nath and E. Boldyreva, Mechanically responsive molecular crystals, Chem. Rev., 2015, 115(22), 12440-12490.

4 C. E. Mulijanto, H. S. Quah, G. K. Tan, B. Donnadieu and J. J. Vittal, Curved crystal morphology, photoreactivity and 
photosalient behaviour of mononuclear $\mathrm{Zn}$ (II) complexes, IUCrJ, 2017, 4(1), 65-71.

5 S. Mittapalli, D. Sravanakumar Perumalla and A. Nangia, Mechanochemical synthesis of $N$-salicylideneaniline: thermosalient effect of polymorphic crystals, IUCrJ, 2017, 4(3), 243-250.

6 Ž. Skoko, S. Zamir, P. Naumov and J. Bernstein, The thermosalient phenomenon. "jumping crystals" and crystal chemistry of the anticholinergic agent oxitropium bromide, J. Am. Chem. Soc., 2010, 132(40), 14191-14202.

7 I. Lončarić, J. Popović, V. Despoja, S. Burazer, I. Grgičević, D. Popović and Ž. Skoko, Reversible thermosalient effect of $N^{\prime}$-2-propylidene-4-hydroxybenzohydrazide accompanied by an immense negative compressibility: structural and theoretical arguments aiming toward the elucidation of jumping phenomenon, Cryst. Growth Des., 2017, 17(8), 4445-4453.

8 D. P. Karothu, J. Weston, I. T. Desta and P. Naumov, Shapememory and self-healing effects in mechanosalient molecular crystals, J. Am. Chem. Soc., 2016, 138(40), 1329813306.

9 A. Khalil, E. Ahmed and P. Naumov, Metal-coated thermosalient crystals as electrical fuses, Chem. Commun., 2017, 53(60), 8470-8473.

10 A. Khalil, C. T. Hu and P. Naumov, Nanoscale crystallization and thermal behaviour of 1,2,4,5-tetrabromobenzene, CrystEngComm, 2018, 20(5), 636-642.

11 J.-H. Ko, K.-S. Lee, S. Chandra Sahoo and P. Naumov, Isomorphous phase transition of 1,2,4,5tetrabromobenzene jumping crystals studied by Brillouin light scattering, Solid State Commun., 2013, 173, 46-50.

12 B. A. Zakharov, A. A. L. Michalchuk, C. A. Morrison and E. V. Boldyreva, Anisotropic lattice softening near the structural phase transition in the thermosalient crystal 1,2,4,5-tetrabromobenzene, Phys. Chem. Chem. Phys., 2018, 20(13), 8523-8532.

13 S. C. Sahoo, S. B. Sinha, M. S. R. N. Kiran, U. Ramamurty, A. F. Dericioglu, C. M. Reddy and P. Naumov, Kinematic and mechanical profile of the self-actuation of thermosalient crystal twins of 1,2,4,5-tetrabromobenzene: A molecular crystalline analogue of a bimetallic strip, $J$. Am. Chem. Soc., 2013, 135(37), 13843-13850.

14 D. K. P. Ng, Y.-O. Yeung, W. K. Chan and S.-C. Yu, Columnar liquid crystals based on 2,3-naphthalocyanine core, Tetrahedron Lett., 1997, 38(38), 6701-6704.

15 L. Chen, Y. Honsho, S. Seki and D. Jiang, Light-harvesting conjugated microporous polymers: Rapid and highly efficient flow of light energy with a porous polyphenylene framework as antenna, J. Am. Chem. Soc., 2010, 132(19), 6742-6748.

16 D. Mondieig, M. A. Cuevas-Diarte and Y. Haget, Polymorphism of tetrachloro-1,2,4,5 benzene and tetrabromo-1,2,4,5 benzene, J. Therm. Anal. Calorim., 1989, 35(7), 2491-2500.

17 G. Gafner, The crystal and molecular structures of overcrowded halogenated compounds. II. $\beta-1: 2-4: 5-$ tetrabromobenzene, Acta Crystallogr., 1960, 13(9), 706-716.
18 F. B. Johnson, Phase-Change in 1,2,4,5-tetrabromobenzene investigated by pure quadrupole resonance, Nature, 1956, 178, 590.

19 S. Krafczyk, H. Jacobi and H. Follner, Twinning of crystals as a result of differences between symmetrical and energetical most favorable structure arrangements, Cryst. Res. Technol., 1994, 29(5), 623-631.

20 H. F. Lieberman, R. J. Davey and D. M. T. Newsham, Br $\cdots B r$ and $\mathrm{Br} \cdots \mathrm{H}$ interactions in action: Polymorphism, hopping, and twinning in 1,2,4,5-tetrabromobenzene, Chem. Mater., 2000, 12(2), 490-494.

21 A. A. Mrse, Y. Lee, P. L. Bryant, F. R. Fronczek, L. G. Butler and L. S. Simeral, Pulsed ${ }^{81} \mathrm{Br}$ nuclear quadrupole resonance spectroscopy of brominated flame retardants and associated polymer blends, Chem. Mater., 1998, 10(5), 1291-1300.

22 G. Gafner, The crystal and molecular structures of overcrowded halogenated compounds. V. $\gamma-1,2: 4,5-$ Tetrabromobenzene, Acta Crystallogr., 1964, 17(8), 982-985.

23 T. Dove Martin, Review: Theory of displacive phase transitions in minerals, Am. Mineral., 1997, 82, 213.

24 K. R. Morris, U. J. Griesser, C. J. Eckhardt and J. G. Stowell, Theoretical approaches to physical transformations of active pharmaceutical ingredients during manufacturing processes, Adv. Drug Delivery Rev., 2001, 48(1), 91-114.

25 J. Anwar and D. Zahn, Polymorphic phase transitions: Macroscopic theory and molecular simulation, Adv. Drug Delivery Rev., 2017, 117, 47-70.

26 R. Dovesi, A. Erba, R. Orlando, C. M. Zicovich-Wilson, B. Civalleri, L. Maschio, M. Rérat, S. Casassa, J. Baima, S. Salustro and B. Kirtman, Quantum-mechanical condensed matter simulations with CRYSTAL, Wiley Interdiscip. Rev.: Comput. Mol. Sci., 2018, e1360.

27 J. P. Perdew, K. Burke and M. Ernzerhof, Generalized gradient approximation made simple, Phys. Rev. Lett., 1996, 77(18), 3865-3868.

28 F. Weigend and R. Ahlrichs, Balanced basis sets of split valence, triple zeta valence and quadruple zeta valence quality for $\mathrm{H}$ to $\mathrm{Rn}$ : Design and assessment of accuracy, Phys. Chem. Chem. Phys., 2005, 7(18), 3297-3305.

29 S. Grimme, J. Antony, S. Ehrlich and H. Krieg, A consistent and accurate $a b$ initio parametrization of density functional dispersion correction (DFT-D) for the 94 elements H-Pu, J. Chem. Phys., 2010, 132(15), 154104.

30 S. Grimme, A. Hansen, J. G. Brandenburg and C. Bannwarth, Dispersion-corrected mean-field electronic structure methods, Chem. Rev., 2016, 116(9), 5105-5154.

31 S. Grimme, S. Ehrlich and L. Goerigk, Effect of the damping function in dispersion corrected density functional theory, $J$. Comput. Chem., 2011, 32, 1456-1465.

32 S. F. Boys and F. Bernardi, The calculation of small molecular interactions by the differences of separate total energies. Some procedures with reduced errors, Mol. Phys., 1970, 19(4), 553-566.

33 L. Maschio, B. Kirtman, M. Rerat, R. Orlando and R. Dovesi, $A b$ initio analytical Raman intensities for periodic systems through a coupled perturbed Hartree-Fock/Kohn-Sham 
method in an atomic orbital basis. I. Theory, J. Chem. Phys., 2013, 139(16), 164101/1-164101/13.

34 L. Maschio, B. Kirtman, M. Rerat, R. Orlando and R. Dovesi, $A b$ initio analytical Raman intensities for periodic systems through a coupled perturbed Hartree-Fock/Kohn-Sham method in an atomic orbital basis. II. Validation and comparison with experiments, J. Chem. Phys., 2013, 139(16), 164102/1-164102/9.

35 D. G. Anderson, Iterative procedures for nonlinear integral equations, J. Assoc. Comput. Mach., 1965, 12(4), 547-560.
36 K. M. White and C. J. Eckhardt, Single crystal Raman spectra of 1,2,4,5-tetrabromobenzene: Calculational and experimental assignment of the internal modes, J. Chem. Phys., 1998, 109(1), 208-213.

37 E. Burgos and H. Bonadeo, The lattice vibrations of 1,2,4,5tetrabromobenzene, Chem. Phys. Lett., 1978, 57, 125-127.

38 F. Shimizu, Y. Suzuki, K. Mitarai, M. Fujino, H. Kawano, Y. Nibu, H. Shimada and R. Shimada, Pressure-induced phase transition in the 1,2,4,5-tetrabromobenzene crystal, Bull. Chem. Soc. Jpn., 1995, 68, 1883-1888. 